\title{
Evolution of gene auto-regulation in the presence of noise
}

\author{
Abhyudai Singh \\ Department of Chemistry \& Biochemistry \\ University of California, San Diego \\ La Jolla, CA 92093 \\ Email: a2singh@ucsd.edu
}

\author{
João Pedro Hespanha \\ Department of Electrical Engineering \\ University of California, Santa Barbara \\ Santa Barbara, CA 93101 \\ Email: hespanha@ece.ucsb.edu
}

\begin{abstract}
Auto-regulatory negative feedback loops, where the protein expressed from a gene inhibits its own expression are common gene network motifs within cells. We investigate when will introducing a negative feedback mechanism be beneficial in terms of increasing a fitness function that is given by the probability of maintaining protein numbers above a critical threshold. Our results show the existence of a trade-off as introducing feedback decreases the average number of protein molecules driving this number closer to the critical threshold (which decreases fitness) but also reduces stochastic fluctuations around the mean (which increases fitness).

We provide analytical conditions under which a negative feedback mechanism can evolve, i.e., introducing feedback will increase the above fitness. Analyses of these conditions show that negative feedbacks are more likely to evolve when (i) the source of noise in the protein population is extrinsic (i.e., noise is caused by fluctuations in exogenous signals driving the gene network) and not intrinsic (i.e., the randomness associated with mRNA/protein expression and degradation); (ii) the dynamics of the exogenous signal causing extrinsic noise is slower than the protein dynamics; and (iii) the critical threshold level for the protein number is low. We also show that $\mathrm{mRNA} /$ protein degradation rates are critical factors in determining whether transcription or translational negative feedback should evolve. In particular, when the mRNA half-life is much shorter than the protein's half-life, then a transcriptional negative feedback mechanism is more likely to evolve. On the other hand, a translational negative feedback mechanism is preferred with more stable mRNAs that have long half-lifes.
\end{abstract}




\section{INTRODUCTION}

The inherent stochastic nature of gene expression processes such as transcription and translation, coupled with low molecule counts of mRNAs and proteins, can lead to large statistical fluctuations in molecule numbers [1], [2], [3], [4], [5]. Evidence is accumulating that these stochastic fluctuations are subject to natural selection and many gene networks have indeed evolved to minimize noise in protein levels [6], [7]. A common gene network motif that has been associated with noise reduction is transcriptional negative feedback, where the protein inhibits the transcription of its own gene [8], [9]. Both theoretical and experimental studies have shown that this form of feedback can reduce stochastic fluctuations in the protein population [10], [11], [12], [13], [14], [15], [16], [17]. In addition, recent work has demonstrated the existence of negative feedback loops at the translational level, where the protein inhibits the translation of its own mRNA. In fact, [18] argued that a translational negative feedback is more efficient in attenuating noise than a transcriptional negative feedback.

In order to counteract the effects of noisy gene expression, we investigate when will introducing a negative feedback mechanisms be beneficial in terms of increasing a certain fitness function. We consider a very simple, yet biologically relevant fitness function, which is given by the probability of maintaining the protein numbers above a critical level. This fitness function is appropriate for various essential proteins whose populations have to be maintained above a threshold for normal cellular functioning. Stochastic fluctuations in the protein population that drive their numbers below this threshold are assumed to compromise the cell's viability. This type of threshold-like effect has been reported for various prokaryotic transcription factors [19]. This fitness function is also appropriate for genes that have stable ON and OFF states corresponding to different environmental inputs (for example, the lambda repressor gene in the lambda phage gene network [20] and the Gal80 gene in the galactose signaling network [21]). For such genes, minimizing random stochastic transitions from the $\mathrm{ON}$ to the OFF state correspond to maximizing the probability of having protein numbers above a critical threshold.

Our motivation for studying the above problem comes from evolution, where negative feedback is introduced through random mutations in the gene sequence and will only persist if there is a positive change in fitness. The above fitness function shows that there is a non-trivial trade-off in introducing a negative feedback mechanism. In particular, introducing negative feedback decreases the average number of protein molecules, which tends to decrease the probability of having protein numbers larger than the 
threshold and hence decreases fitness. However, negative feedback also reduces fluctuations in protein numbers about the mean, making it less likely that the protein count will fall below the critical threshold. If the net change in fitness is positive, then negative feedback is said to be evolvable.

We perform a systematic analysis of this trade-off and provide explicit analytical conditions under which negative feedback mechanisms are evolvable. In particular, we consider feedbacks both at the transcriptional and translational level. We show that both these feedback mechanisms are more evolvable when noise in the protein numbers comes form a slowly varying noisy exogenous signal that drives gene expression (i.e., source of protein noise is extrinsic) and not from the inherent stochasticity of mRNA/protein expression and degradation. This latter form of noise is often referred to in literature as intrinsic noise. Finally, we compare transcriptional negative feedback with a translational negative feedback and show that the ratio $\frac{\mathrm{mRNA} \text { half-life }}{\text { protein half-life }}$ is the critical factor in deciding which feedback mechanism is more likely to evolve.

\section{FITNESS FUNCTION}

We consider a generic protein $\mathrm{X}$ that must be maintained above a above a critical threshold $T$. Fluctuations in the population that cause the protein numbers to drift below this threshold prevent it to perform its biological function. The probability that the protein count $x$ is larger than $T$ is given by $f(\bar{x}, \sigma):=$ Probability $\{T<x\}$, the complement of the cumulative density function evaluated at the critical threshold, and is assumed to be completely determined by the steady-state average protein count $\bar{x}$ and its standard deviation $\sigma$. For example, if the steady-state distribution of the protein count $x$ is approximately Gaussian, then

$$
f(\bar{x}, \sigma)=\frac{1}{2}\left(1+\frac{2}{\sqrt{\pi}} \int_{y=0}^{\frac{\bar{x}-T}{\sqrt{2} \sigma}} \exp \left(-y^{2}\right) d y\right) .
$$

Since this above probability should be high, we also assume that the threshold $T$ is smaller than the mean protein count $\bar{x}$.

In order to study the effect of feedback on $f(\bar{x}, \sigma)$, it will be convenient to work with a normalized fitness $F$ defined by

$$
F:=\frac{f(\bar{x}, \sigma)}{f\left(\bar{x}_{n f}, \sigma_{n f}\right)}
$$


where $\bar{x}_{n f}, \sigma_{n f}$ represent the steady-state mean and the standard deviation of the protein count, respectively, in the absence of feedback. In general, this normalized fitness will satisfy the following properties:

$$
\frac{\partial F}{\partial \bar{x}}>0, \quad \frac{\partial F}{\partial \sigma}<0
$$

This is because for a fixed $\sigma$, decreasing $\bar{x}$ should decrease fitness as it will drive the protein count closer to the critical threshold. On the other hand, for a fixed $\bar{x}$, increasing $\sigma$ should also decrease fitness as then the protein count is more likely to fall below the threshold by random chance. In the next section we introduce a transcriptional negative feedback model and derive analytical expressions for the steady-state moments of $x$.

\section{TRANSCRIPTIONAL NEGATIVE FEEDBACK MODEL}

We consider a model for an auto-regulatory negative feedback mechanism at the transcriptional level, where the protein inhibits the transcription of its own mRNA. This model considers three chemical species: the protein $\mathrm{X}$, its transcribed mRNA denoted by $\mathrm{M}$, and an exogenous species $\mathrm{Z}$ that drives the transcription process. In the sequel, we use the corresponding small letters $z(t), m(t)$ and $x(t)$ to denote the number of molecule of the corresponding species at time $t$. The symbols $\bar{z}, \bar{m}, \bar{x}$ represent the steady-state average count of the corresponding species, respectively. A summary of the notation used in this paper is provided in Table I. These species are involved in the following set of six chemical reactions:

$$
\begin{array}{ll}
* \stackrel{k_{\text {exogenous }}}{\longrightarrow} \mathrm{Z}, \mathrm{Z} \stackrel{k_{\text {degexo }}}{\longrightarrow} *, & \text { (exogenous signal production \& degradation) } \\
* \stackrel{k_{\text {transcription }} g_{1}(x, z)}{\longrightarrow} \mathrm{M}, \mathrm{M} \stackrel{k_{\text {degmRNA }}}{\longrightarrow} *, & \text { (mRNA transcription \& degradation) } \\
\mathrm{M} \stackrel{k_{\text {translation }}}{\longrightarrow} \mathrm{M}+\mathrm{X}, \mathrm{X} \stackrel{k_{\text {degpro }}}{\longrightarrow} *, & \text { (protein translation \& degradation) }
\end{array}
$$

The first two reactions in (4) represent the dynamics of the exogenous signal Z, which is assumed to be

produced at a rate $k_{\text {exogenous }}$ and degrades at a constant rate $k_{\text {degexo }}$. This results in a Poisson distribution for $z$ with steady-state level and standard deviation given by

$$
\bar{z}=\frac{k_{\text {exogenous }}}{k_{\text {degexo }}}, \quad \sigma_{\text {exogenous }}=\sqrt{\bar{z}}
$$

respectively. The quantity $\sigma_{\text {exogenous }}$ represents the amount of stochastic fluctuations that enters the autoregulatory gene network through the exogenous signal $z$. 
TABLE I

A SUMMARY OF THE NOTATION USED IN THIS PAPER.

\begin{tabular}{|c|c|}
\hline$z(t), m(t), x(t)$ & $\begin{array}{l}\text { Species count of the exogenous signal, mRNA and the } \\
\text { protein, respectively, at time } t\end{array}$ \\
\hline $\bar{z}, \sigma_{\text {exogenous }}$ & Steady-state average and standard deviation of exogenous signal $z$ \\
\hline $\bar{m}, \bar{x}$ & $\begin{array}{c}\text { Steady-state average species count of the mRNA and } \\
\text { the protein, respectively }\end{array}$ \\
\hline $\bar{m}_{n f}, \bar{x}_{n f}$ & $\begin{array}{l}\text { Steady-state average species count of the mRNA and } \\
\text { the protein, respectively, when there is no feedback }\end{array}$ \\
\hline$\sigma, \sigma_{i n t}, \sigma_{e x t}$ & $\begin{array}{l}\text { Steady-state standard deviation of } x \text {, its intrinsic and extrinsic } \\
\text { component, respectively }\end{array}$ \\
\hline$\sigma_{n f}, \sigma_{i n t-n f}, \sigma_{e x t-n f}$ & $\begin{array}{l}\text { Steady-state standard deviation of } x \text {, its intrinsic and extrinsic } \\
\text { component, respectively, when there is no feedback }\end{array}$ \\
\hline$F$ & Normalized fitness function given by $\frac{f(\bar{x}, \sigma)}{f\left(\bar{x}_{n f}, \sigma_{n f}\right)}$ \\
\hline$P, Q$ & $\begin{array}{l}\text { Measure how sensitive } F \text { is to changes in the steady-state } \\
\text { protein mean } \bar{x} \text { and standard deviation } \sigma \text {, respectively }\end{array}$ \\
\hline$H, a$ & Hill coefficient and strength of the negative feedback, respectively \\
\hline$T, \gamma$ & $\begin{array}{l}\text { Critical and normalized threshold, respectively, below } \\
\text { which protein is functionally in-active }\end{array}$ \\
\hline$k_{\text {degexo }}, k_{\text {degmRNA }}, k_{\text {degpro }}$ & Exogenous signal, mRNA and protein degradation rate, respectively \\
\hline$\kappa$ & $\begin{array}{l}k_{\text {degexo }} / k_{\text {degpro }}, \text { measure of how fast the protein } \\
\text { dynamics is compared to the exogenous signal dynamics }\end{array}$ \\
\hline$\varepsilon$ & $\begin{array}{l}k_{\text {degpro }} / k_{\text {degmRNA }}, \text { measure of how fast the mRNA } \\
\text { dynamics is compared to the protein dynamics }\end{array}$ \\
\hline$\eta$ & $\bar{x}_{n f} / \bar{m}_{n f}$ \\
\hline$H_{\text {int-transcription }}, H_{\text {ext-transcription }}$ & $\begin{array}{l}\text { Critical level of } H \text {, above which a transcriptional feedback is } \\
\text { evolvable, when intrinsic and extrinsic noise dominate, respectively }\end{array}$ \\
\hline$H_{\text {int-translation }}, H_{\text {ext-translation }}$ & $\begin{array}{l}\text { Critical level of } H \text {, above which a translational feedback is } \\
\text { evolvable, when intrinsic and extrinsic noise dominate, respectively }\end{array}$ \\
\hline
\end{tabular}


The mRNA transcription rate is given by $k_{\text {transcription }} g_{1}(x, z)$ and monotonically decreases with increasing protein count $x$. This protein-dependent transcription rate mediates a negative feedback mechanism as any increase in protein count by random chance is compensated by a decrease in transcriptional activity. The function $g_{1}(x, z)$ is given by the well known Hill equation

$$
g_{1}(x, z)=\frac{g(z)}{1+(a x)^{H}}
$$

where $H \geq 1$ denotes the Hill coefficient and the constant $a$ is the negative feedback strength. The exogenous signal enters the transcription rate through the function $g(z)$. Depending on whether $z$ is an activator or repressor, $g(z)$ would be a monotonically increasing or decreasing function, respectively. Note that $a=0$ corresponds to no negative feedback in gene expression. This is because when $a=0$ the transcription rate is simply given by

$$
\left.k_{\text {transcription }} g_{1}(x, z)\right|_{a=0}=k_{\text {transcription }} g(z) \text {, }
$$

which is independent of the protein count. Finally, we assume that mRNA degradation, protein translation from the mRNA, and protein degradation occur at constant rates $k_{\text {degmRNA }}, k_{\text {translation }}$ and $k_{\text {degpro }}$, respectively.

Using a linear approximation for the transcription rate $g_{1}(x, z)$ we derive approximate expressions for $\bar{x}$ and $\sigma$ in Appendix A. These expression are valid in the limit when stochastic fluctuations in species count are small in comparison to their mean values, which is likely in proteins that need to be regulated. In particular, the steady-state average number of protein molecules $\bar{x}$ and mRNA molecules $\bar{m}$ satisfy the following equations

$$
\frac{\bar{x}}{\bar{x}_{n f}}=\frac{1}{1+(a \bar{x})^{H}}<1, \quad \frac{\bar{m}}{\bar{m}_{n f}}=\frac{\bar{x}}{\bar{x}_{n f}}<1
$$

where

$$
\bar{x}_{n f}=\frac{k_{\text {transcription }} k_{\text {translation }}}{k_{\text {degmRNA }} k_{\text {degpro }}} g(\bar{z}), \quad \bar{m}_{n f}=\frac{k_{\text {transcription }}}{k_{\text {degmRNA }}} g(\bar{z}),
$$

are the steady-state average protein and mRNA counts, respectively, when there is no feedback. The 
steady-state standard deviation of $x$ is given by

$$
\begin{gathered}
\sigma=\sqrt{\sigma_{\text {int }}^{2}+\sigma_{\text {ext }}^{2}} \\
\sigma_{\text {int }}=\sqrt{\bar{x} \frac{1+\varepsilon(1-S)+\varepsilon \eta}{(1-S)(1+\varepsilon)}}, \quad \sigma_{\text {ext }}=\left.\frac{d g(z)}{d z}\right|_{z=\bar{z}} \frac{\bar{x} \sigma_{\text {exogenous }}}{g(\bar{z})} \sqrt{\frac{1+\mathcal{E}+\mathcal{E} \kappa}{(1-S)(1+\varepsilon)[(1+\kappa)(1+\varepsilon \kappa)-S]}}
\end{gathered}
$$

where

$$
S=\left.\frac{\bar{x}}{g_{1}(\bar{x}, \bar{z})} \frac{d g_{1}(x, \bar{z})}{d x}\right|_{x=\bar{x}}<0, \quad \eta=\frac{\bar{x}_{n f}}{\bar{m}_{n f}}, \quad \varepsilon=\frac{k_{\text {degpro }}}{k_{\text {degmRNA }}}, \quad \kappa=\frac{k_{\text {degexo }}}{k_{\text {degpro }}}
$$

are dimensionless constants. In the above formula, $\sigma_{\text {int }}$ denotes the intrinsic component of $\sigma$ and represents the stochastic fluctuations in the protein level due to random transcription, translation and protein/mRNA degradation events. On the other hand, $\sigma_{\text {ext }}$ denotes the extrinsic component of $\sigma$ and corresponds to protein fluctuations due to the noisy exogenous signal driving gene expression [22], [23], [24]. The dimensionless constant $\varepsilon$ is a measure of how fast the mRNA dynamics is compared to the protein dynamics, and $\kappa$ is a measure of how fast the protein dynamics is compared to the exogenous signal dynamics. In the absence of feedback $a=S=0$ and (10) reduces to

$$
\begin{gathered}
\sigma_{n f}=\sqrt{\sigma_{\text {int }-n f}^{2}+\sigma_{\text {ext }-n f}^{2}} \\
\sigma_{\text {int }-n f}=\sqrt{\bar{x}_{n f} \frac{1+\varepsilon+\varepsilon \eta}{1+\varepsilon}}, \quad \sigma_{\text {ext }-n f}=\left.\frac{d g(z)}{d z}\right|_{z=\bar{z}} \frac{\bar{x}_{n f} \sigma_{\text {exogenous }}}{g(\bar{z})} \sqrt{\frac{1+\mathcal{E}+\varepsilon \kappa}{(1+\varepsilon)(1+\kappa)(1+\varepsilon \kappa)}}
\end{gathered}
$$

These expressions represent the steady-state standard deviation in $x$, and its intrinsic and extrinsic components, when there is no negative feedback.

Figure 1 shows how $\bar{x}$ and $\sigma$ change as we increase the negative feedback strength $a$ from an initial value of zero. Increasing the feedback strength from zero decreases the average protein number $\bar{x}$ from $\bar{x}_{n f}$, which causes $F$ to decrease [see (3)]. On the other hand, the standard deviation $\sigma$ about the mean also decrease with increasing feedback strength which causes $F$ to increase. If the net change in the fitness is positive then the negative feedback is said to be evolvable. Our goal in the next section is to analyze under what conditions and for what range of parameters is a transcriptional negative feedback mechanism evolvable. 


\section{EVOLVABILITY OF A TRANSCRIPTIONAL NEGATIVE FEEDBACK}

Introducing feedback (i.e., increasing the negative feedback strength from zero) will increase the normalized fitness $F$, if and only if,

$$
\left.\frac{d F}{d a^{H}}\right|_{a=0}>0
$$

Using (3), the above inequality can be written as

$$
\left.\frac{P}{\bar{x}_{n f}} \frac{d \bar{x}}{d a^{H}}\right|_{a=0}>\left.\frac{Q}{\sigma_{n f}} \frac{d \sigma}{d a^{H}}\right|_{a=0}
$$

where

$$
P=\left.\bar{x}_{n f} \frac{\partial F}{\partial \bar{x}}\right|_{a=0}>0, \quad Q=-\left.\sigma_{n f} \frac{\partial F}{\partial \sigma}\right|_{a=0}>0
$$

are positive dimensionless constants that measure how sensitive fitness $F$ is to changes in the steady-state protein mean and standard deviation, respectively.

Using the steady-state moments derived in the previous section we conclude that (14) holds, if and only if,

$$
H>H_{\text {transcription }}=\left\{\begin{array}{lll}
H_{\text {int-transcription }}=\frac{1+\varepsilon+\varepsilon \eta}{1+\varepsilon \eta} \frac{2 P-Q}{Q} & \text { when } & \sigma_{\text {int }} \gg \sigma_{\text {ext }} \\
H_{\text {ext-transcription }}=\frac{2(1+\kappa)(1+\varepsilon \kappa)}{2+\kappa+\varepsilon \kappa+\varepsilon \kappa^{2}} \frac{P-Q}{Q} & \text { when } & \sigma_{\text {int }} \ll \sigma_{\text {ext }} .
\end{array}\right.
$$

where $H_{\text {transcription }}$ is a critical value of Hill coefficient above which transcriptional negative feedback is evolvable. In the regime where intrinsic (extrinsic) noise dominates $\sigma$, the constant $H_{\text {transcription }}$ reduces to $H_{\text {int-transcription }}\left(H_{\text {ext-transcription }}\right)$. Note that if $P$ is sufficiently small (this implies form (15) that $F$ is not sensitive to changes in the mean protein level), then $H_{\text {transcription }}<0$ and negative feedback is always evolvable. However, for most physiologically relevant protein distributions $H_{\text {transcription }}$ is positive (see (19)), in which case a negative feedback mechanism can only evolve if the Hill coefficient of the feedback is sufficiently large. In the sequel, we focus on this latter case where feedback is evolvable only for a range of parameters given by $H>H_{\text {transcription }}>0$. In particular, feedback is said to be more evolvable if the gene network has a smaller value of $\left.H_{\text {transcription. As }} \frac{d F}{d a^{H}}\right|_{a=0}$ is proportional to the difference

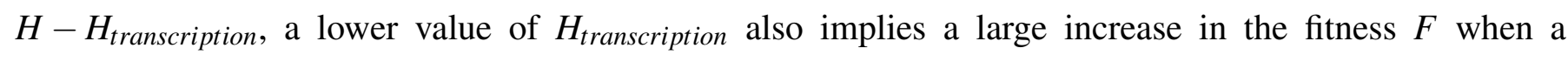
negative feedback mechanism is introduced. We next investigate what factors make negative feedback 
more evolvable, i.e., lead to lower values of $H_{\text {transcription }}$.

An analysis of (16) reveals that $H_{\text {transcription }}$ is lower when extrinsic noise dominates $\sigma$ than when intrinsic noise dominates, i.e., $H_{\text {int-transcription }}>H_{\text {ext-transcription }}$. Thus for Hill coefficients $H>H_{\text {int-transcription }}$, negative feedback is always evolvable irrespective of whether noise in protein population is intrinsic or extrinsic. However, if $H$ is in the range $H_{\text {ext-transcription }}<H<H_{\text {int-transcription, }}$ then negative feedback can only evolve if there is significant extrinsic noise in the protein population. We illustrate this point in more detail by assuming that the protein distribution is approximately Gaussian, in which case we have from (1) and (15) that

$$
\frac{P}{Q}=\frac{1}{1-\gamma}
$$

where the constant $\gamma$ is a normalized threshold defined by

$$
\gamma=\frac{T}{\bar{x}_{n f}}<1
$$

Substituting (17) in (16) the evolvability condition reduces to

$$
H>H_{\text {transcription }}=\left\{\begin{array}{lrr}
H_{\text {int-transcription }}=\frac{1+\varepsilon+\varepsilon \eta}{1+\varepsilon \eta} \frac{1+\gamma}{1-\gamma}>1 & \text { when } \quad \sigma_{\text {int }} \gg \sigma_{\text {ext }} \\
H_{\text {ext-transcription }}=\frac{2(1+\kappa)(1+\varepsilon \kappa)}{2+\kappa+\varepsilon \kappa+\varepsilon \kappa^{2}} \frac{\gamma}{1-\gamma}>0 & \text { when } \quad \sigma_{\text {int }} \ll \sigma_{\text {ext }} .
\end{array}\right.
$$

Since in this case $H_{\text {int-transcription }}>1$, we conclude that when fluctuations in protein numbers are mostly due to random protein/mRNA expression and degradation events (intrinsic noise), a negative feedback with a Hill coefficient of one $(H=1)$ is never evolvable. However, when extrinsic noise dominates and the normalized threshold $\gamma$ is small enough such that $H_{\text {ext-transcription }}<1$, then a transcriptional negative feedback with $H=1$ is evolvable (see Figure 2). These results show that a transcriptional negative feedback mechanism is more likely to evolve if the source of protein noise is extrinsic rather than intrinsic.

Note that the critical value of the Hill coefficient $H_{\text {ext-transcription }}$ is a monotonically increasing function of $\kappa=k_{\text {degexo }} / k_{\text {degpro }}$. This suggests that negative feedback is more likely to evolve when $\kappa$ is small, i.e., the exogenous signal dynamics is much slower compared to the protein dynamics (see Figure 3). This figure also shows that the range of evolvable Hill coefficients considerably shrinks with increasing normalized threshold $\gamma$. Based on this we conclude that negative feedback is more likely to evolve when the normalized threshold $\gamma$ is small (i.e., threshold $T$ is much lower than the mean population). 
Finally, the above evolvability conditions show that $H_{\text {transcription }}$ is an increasing function of $\varepsilon$. We recall that $\varepsilon=k_{\text {degpro }} / k_{\text {degmRNA }}$ is a measure of how fast the mRNA dynamics is compared to the protein dynamics, with smaller values of $\varepsilon$ denoting a shorter mRNA half-life compared to the protein half-life. Hence, for all other parameters fixed, as we make the mRNA half-life longer (i.e., as we increase $\varepsilon$ ), the evolvability of a transcriptional feedback decreases. This point is illustrated in Figure 4 which shows that the evolvability region shrinks as we increase $\varepsilon$.

We conclude this section by summarizing its main results: Any of the following conditions increases the evolvability of transcriptional negative feedback:

1) Stochastic fluctuations in the protein count are dominated by extrinsic noise, rather than intrinsic noise

2) Dynamics of the exogenous signal much slower than the protein dynamics

3) mRNA dynamics much faster than the protein dynamics

4) A small normalized threshold.

\section{EvolvabiLITY OF A TRANSLATIONAL NEGATIVE FEEDBACK}

We now derive evolvability conditions for a translational feedback mechanism and contrast them with evolvability conditions for transcriptional feedback.

\section{A. Translational negative feedback model}

Our translational negative feedback model involves the same basic set of species, but now the protein population $x$ affects the protein translation rate instead of the mRNA transcription rate, which is captured by the following set of chemical reactions:

$$
\begin{array}{ll}
* \stackrel{k_{\text {exogenous }}}{\longrightarrow} \mathrm{Z}, \mathrm{Z} \stackrel{k_{\text {degexo }}}{\longrightarrow} *, & \text { (exogenous signal production \& degradation) } \\
* \stackrel{k_{\text {transcription }} g(z)}{\longrightarrow} \mathrm{M}, \mathrm{M} \stackrel{k_{\text {degmRNA }}}{\longrightarrow} *, & \text { (mRNA transcription \& degradation) } \\
\mathrm{M} \stackrel{k_{\text {translation }} g_{2}(x)}{\longrightarrow} \mathrm{M}+\mathrm{X}, \mathrm{X} \stackrel{k_{\text {degpro }}}{\longrightarrow} *, & \text { (protein translation \& degradation) }
\end{array}
$$

where

$$
g_{2}(x)=\frac{1}{1+(a x)^{H}} .
$$


As before $H$ and $a$ denote the Hill coefficient and the strength of the negative feedback, respectively. Note that unlike the previous model in (4), the transcription rate is now a constant independent of $x$ and the translation rate monotonically decreases with increasing protein count.

\section{B. Evolvability conditions}

The analysis in Appendix B shows that for translational feedback, the steady-state average protein and mRNA counts are given by

$$
\frac{\bar{x}}{\bar{x}_{n f}}=\frac{1}{1+(a \bar{x})^{H}}<1, \quad \frac{\bar{m}}{\bar{m}_{n f}}=1
$$

with following steady-state protein standard deviation

$$
\begin{gathered}
\sigma=\sqrt{\sigma_{\text {int }}^{2}+\sigma_{\text {ext }}^{2}} \\
\sigma_{\text {int }}=\sqrt{\bar{x} \frac{1+\varepsilon(1-S)+\varepsilon \eta \bar{x} / \bar{x}_{n f}}{(1-S)[1+(1-S) \varepsilon]}}, \quad \sigma_{\text {ext }}=\left.\frac{d g(z)}{d z}\right|_{z=\bar{z}} \frac{\bar{x} \sigma_{\text {exogenous }}}{g(\bar{z})} \sqrt{\frac{1+\varepsilon(1-S+\kappa)}{(1-S)[1+(1-S) \varepsilon](1-S+\kappa)(1+\varepsilon \kappa)}}
\end{gathered}
$$

Using (12), (13), (22), (23) we conclude that translational negative feedback is evolvable, if and only if,

$$
H>H_{\text {translation }}= \begin{cases}H_{\text {int-translation }}=\frac{(1+\varepsilon)[2(1+\varepsilon+\varepsilon \eta) P / Q-(1+\varepsilon+2 \varepsilon \eta)]}{1+\varepsilon(2+\varepsilon+\eta+2 \varepsilon \eta)} & \text { when } \quad \sigma_{\text {int }} \gg \sigma_{\text {ext }} \\ H_{\text {ext-translation }}=\frac{2(1+\kappa)(1+\varepsilon)(1+\varepsilon+\varepsilon \kappa)}{2+\kappa+4 \varepsilon+2 \varepsilon^{2}+4 \varepsilon \kappa+4 \varepsilon^{2} \kappa+\varepsilon \kappa^{2}+2 \varepsilon^{2} \kappa^{2}} \frac{P-Q}{Q} & \text { when } \quad \sigma_{\text {int }} \ll \sigma_{\text {ext }}\end{cases}
$$

where $H_{\text {translation }}$ represents a critical level of Hill coefficient above which a translational feedback can evolve. As done in the previous section, assuming $H_{\text {translation }}>0$, we investigate what factors increase the range of parameters for which feedback is evolvable. An analysis of (24) reveals that $H_{\text {ext-translation }}<$ $H_{\text {int-translation }}$ and $H_{\text {ext-translation }}$ is an increasing function of $\kappa$. Hence, like transcriptional feedback, translational feedback is also more likely to evolve if the noise in the protein count arises from a slowly varying noisy exogenous signal. However, in contrast to transcriptional feedback, $H_{\text {translation }}$ is a decreasing function of $\varepsilon$. Hence, as we make the mRNA half-life longer (i.e., as we increase $\varepsilon$ ), the evolvability of a translational feedback increases (see Figure 5 and contrast it with Figure 4).

\section{Comparing evolvability conditions for transcriptional and translational feedback as a function of $\varepsilon$}

In gene networks, the mRNA half-life is generally much smaller than the protein half-life [25]. This

corresponds to low values of the dimensionless constant $\varepsilon=\frac{k_{\text {degpro }}}{k_{\text {degmRNA }}}$. Comparing (24) with (16) we see 
that

$$
\begin{aligned}
& \lim _{\varepsilon \rightarrow 0} H_{\text {int-transcription }}=\lim _{\varepsilon \rightarrow 0} H_{\text {int-translation }}=\frac{2 P-Q}{Q}, \\
& \lim _{\varepsilon \rightarrow 0} H_{\text {ext-transcription }}=\lim _{\varepsilon \rightarrow 0} H_{\text {ext-translation }}=\frac{2(1+\kappa)}{2+\kappa} \frac{P-Q}{Q} .
\end{aligned}
$$

Hence, in the biologically relevant regime of small $\varepsilon$, both transcriptional and translational feedback are equally likely to evolve. As we now increase $\varepsilon$ from zero, the evolvability of a transcriptional feedback decreases while the evolvability of a translational feedback increases. Based on this we conclude that for any non-zero $\varepsilon$, a translational feedback is always more evolvable than a transcriptional feedback and the difference between their evolvabilities increases with increasing $\varepsilon$. This point is illustrated in Figure 6 which plots the ratio

$$
\frac{\text { Fitness with translational feedback }}{\text { Fitness with transcriptional feedback }}
$$

as a function of the feedback strength $a$ for small and large $\varepsilon$. As predicted, when $\varepsilon$ is small then this ratio is close to one, and introducing feedback increases fitness by the same amount for both transcriptional and translational feedback. On the other hand, for higher values of $\varepsilon$ this ratio is much larger than one, in which case introducing a translational feedback mechanism will increase fitness by a much larger amount than introducing a translational feedback mechanism.

As we discuss below, both transcriptional and translational feedback have different levels of energy expenditure. We next compare the evolvabilities of transcriptional and translational feedback by also factoring in the energy costs associated with them.

\section{Energy consumption}

For a fixed negative feedback strength $a$, both transcriptional and translation feedback have the same steady-state mean protein count given by

$$
\frac{\bar{x}}{\bar{x}_{n f}}=\frac{1}{1+(a \bar{x})^{H}}<1
$$

Hence, the energy costs of protein production is identical in these two different feedback mechanisms. However, these feedbacks have different costs associated with mRNA production. This is because for a given negative feedback strength, a transcriptional feedback mechanism has a lower mean mRNA count 
than a translational feedback mechanism [see (8) and (22)]. Combining both the costs of mRNA and protein production we conclude that a translational negative feedback has an overall higher steady-state ATP (energy) consumption compared to a transcriptional feedback (see Figure 6).

As mentioned before, when the mRNA dynamics is much faster than the protein dynamics (small $\varepsilon$ ), translational feedback does not provide any advantage in terms of increasing the normalized fitness $F$. Hence, in this physiologically relevant regime, the better energy efficiency of transcriptional feedback loops make them more likely to evolve. However, energy inefficient translational feedback loops can evolve when $\varepsilon$ is large, as in this case they provide a significant advantage over transcriptional feedback in terms of increasing the normalized fitness (see Figure 6).

\section{DISCUSSION}

Auto-regulatory gene networks in which a protein expressed by a gene inhibits its own production are common motifs within cells. Using a fitness function motivated by a threshold like response of the protein population, we considered the evolvability of such auto-regulatory gene networks from a primitive network with no auto-regulation. An analysis based on the linear noise approximation provided explicit analytical conditions under which an auto-regulatory mechanism can evolve. Our conclusion is that introducing negative feedback will increase fitness (as defined in Section II), if and only if, the Hill coefficient of the feedback is sufficiently large. In particular inequalities (16) and (24) hold, respectively, for transcriptional and translational feedback.

\section{A. Intrinsic versus extrinsic noise}

A systematic analysis of these inequalities showed that negative feedback is more likely to evolve when the source of fluctuations in the protein population is mainly due to extrinsic noise inserted in the network due to fluctuations in exogenous signals driving gene expression, rather than the intrinsic noise that arises solely from the random fluctuations due to protein/mRNA expression and degradation. In fact, for both forms of feedback there is a range of values for the Hill coefficient for which feedback cannot involve when intrinsic noise dominates, but for which feedback is evolvable when extrinsic noise dominates. This range of values for the Hill coefficient narrows as the dynamics of the exogenous signal becomes faster. Hence, negative feedback is more likely to evolve when the extrinsic noise in protein numbers arises from a slowly varying noisy exogenous signal. This is consistent with other results in the literature which show 
that negative feedbacks are more effective in reducing the extrinsic component of noise than its intrinsic component [26], [16], [22], [27], [17].

It is important to point out that the above result is entirely based on a model where the exogenous signal is produced and degraded at a constant rate. A consequence of this is that the noise in the exogenous signal mainly comes at low frequencies, which can be easily attenuated through a negative feedback mechanism. However, there may be other sources of exogenous noise that cannot be modeled by such simple dynamics (for example see [28]) and more work is clearly warranted for such cases.

\section{B. Critical protein threshold level}

Figures 3-5 show that increasing the protein threshold $T$ considerably shrinks the range of evolvable Hill coefficients, and makes transcriptional/translational feedback less evolvable. At a qualitative level this arises because high values of $T$ make the fitness function more sensitive to changes in the mean protein count. Recall that introducing a feedback mechanism decreases the mean protein count, which in turn reduce the fitness $F$. When the protein threshold is low (high), the corresponding reduction in fitness when feedback is introduced is smaller (larger), and results in a more (less) evolvable negative feedback.

\section{Translation versus transcriptional negative feedback}

We also illustrated the tradeoffs in introducing transcriptional and translational negative feedback. This tradeoff is best understood if we consider both the benefits and the energy costs of introducing a negative feedback mechanism. In terms of the fitness function $F$, a translational feedback mechanism is always more evolvable, i.e., introducing translational feedback causes a higher increase in $F$ compared to a introducing a transcriptional feedback mechanism. However, these translational feedbacks come at at a higher energy cost.

Our analysis suggests that the critical factor determining whether a transcription or translational feedback

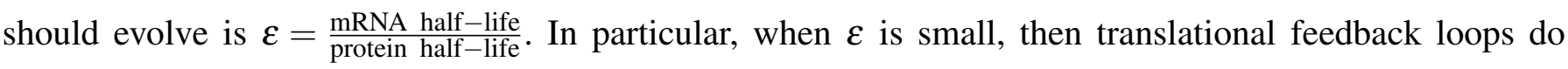
not provide any advantage in terms of increasing $F$ compared to transcriptional feedback loops (see Section V-C). Thus, energy efficient transcriptional feedbacks are more likely to evolve in this case. This result may explain the ubiquity of transcriptional negative feedback mechanisms in gene networks which are known to have short mRNA half lifes and long protein half-lifes. For example, recent studies 
have revealed that nearly $40 \%$ of prokaryotic transcription factors auto-regulate their expression through transcriptional negative feedback [29], [30].

On the other hand, translational feedback loops have a clear advantage when $\varepsilon$ is large. For example, consider a scenario where the mRNA is very stable and has a long half-life. Here, any random increase in mRNA levels above its mean will take a long time to decay away. In a transcriptional negative feedback mechanism, where the rate of protein production from the mRNA is fixed, this results in a transient increase in protein levels that also lasts for a long time. Clearly, transcriptional negative feedbacks are not an effective mechanisms to control protein noise in this case. On the other hand, translational negative feedbacks are very effective in attenuating noise here, as slow mRNA fluctuations about its mean are easily compensated by modulating the protein production rate. In summary, energy inefficient translation feedback loops are more likely to evolve when $\varepsilon$ is large, as in this case they provide much higher value of the fitness $F$ compared to transcriptional negative feedbacks. We illustrate this point with the bacteriophage T4 gene 32 which is known to express an extremely stable mRNA [31]. Consistent with our hypothesis, this gene is negatively auto-regulated at the translational level and not at the transcriptional level [31], [32].

In summary, we have developed results predicting when negative feedback loops are more likely to evolve. Our predictions, although made in the context of the fitness function defined in Section II, would be true at a qualitative level for fitness function that reflects a tradeoff for which decreasing the mean protein count and increasing fluctuations about the mean are detrimental to the cell's viability. Our results not only explain the prevalence of certain design motifs in gene networks but also make explicit predictions that could be experimentally tested. For example, based on our analysis we predict that naturally occurring

gene networks with translation feedback loops should have a higher ratio of $\varepsilon=\frac{\mathrm{mRNA} \text { half-life }}{\text { protein half-life }}$ compared to networks with transcriptional feedback loops.

\section{ACKNOWLEDGMENT}

The authors would like to thank Mustafa Khammash for various discussion on this topic. The authors also acknowledge funding from the Institute for Collaborative Biotechnologies through grant DAAD1903-D-0004 from the U.S. Army Research Office and by the National Science Foundation under Grant No. ECCS-0725485. 


\section{REFERENCES}

[1] M. C. Walters, S. Fiering, J. Eidemiller, W. Magis, M. Groudine, and D. I. K. Martin, "Enhancers increase the probability but not the level of gene expression," Proceedings of the National Academy of Sciences, vol. 92, pp. 7125-7129, 1995.

[2] W. J. Blake, M. Kaern, C. R. Cantor, and J. J. Collins, “Noise in eukaryotic gene expression,” Nature, vol. 422, pp. 633-637, 2003.

[3] M. B. Elowitz, A. J. Levine, E. D. Siggia, and P. S. Swain, "Stochastic gene expression in a single cell," Science, vol. 297, pp. 1183-1186, 2002.

[4] J. M. Raser and E. K. O’Shea, "Noise in gene expression: Origins, consequences, and control," Science, vol. 309, pp. 2010 - 2013, 2005.

[5] J. L. Spudich and D. E. K. Jr, "Non-genetic individuality: chance in the single cell," Nature, vol. 262, pp. 467-471, 1976.

[6] B. Lehner, "Selection to minimise noise in living systems and its implications for the evolution of gene expression," Molecular Systems Biology, 2008.

[7] H. B. Fraser, A. E. Hirsh, G. Giaever, J. Kumm, and M. B. Eisen, "Noise minimization in eukaryotic gene expression,” PLoS Biology, vol. 2, 2004 .

[8] U. Alon, "Network motifs: theory and experimental approaches," Nature Reviews Genetics, vol. 8, pp. 450-461, 2007.

[9] M. E. Wall, W. S. Hlavacek, and M. A. Savageau, "Design principles for regulator gene expression in a repressible gene circuit," J. of Molecular Biology, vol. 332, pp. 861-876, 2003.

[10] M. A. Savageau, "Comparison of classical and autogenous systems of regulation in inducible operons," Nature, vol. 252, pp. 546-549, 1974.

[11] D. Orrell and H. Bolouri, "Control of internal and external noise in genetic regulatory networks," J. of Theoretical Biology, vol. 230, pp. 301-312, 2004.

[12] Y. Tao, X. Zheng, and Y. Sun, "Effect of feedback regulation on stochastic gene expression,” J. of Theoretical Biology, vol. 247, pp. 827-836, 2007.

[13] A. Becskei and L. Serrano, "Engineering stability in gene networks by autoregulation,” Nature, vol. 405, pp. 590-593, 2000.

[14] R. Tomioka, H. Kimura, T. J. Kobayashi, and K. Aihara, "Multivariate analysis of noise in genetic regulatory networks," J. of Theoretical Biology, vol. 229, pp. 501-521, 2004.

[15] M. L. Simpson, C. D. Cox, and G. S. Sayler, "Frequency domain analysis of noise in autoregulated gene circuits," PNAS, vol. 100, pp. 4551-4556, 2003.

[16] Y. Dublanche, K. Michalodimitrakis, N. Kummerer, M. Foglierini, and L. Serrano, "Noise in transcription negative feedback loops: simulation and experimental analysis," Molecular Systems Biology, 2006.

[17] A. Singh and J. Hespanha, “Optimal feedback strength for noise suppression,” Biophysical Journal, To appear, 2009.

[18] P. S. Swain, "Efficient attenuation of stochasticity in gene expression through post-transcriptional control," J. Molecular Biology, vol. 344, pp. 956-976, 2004.

[19] K. Sneppen, M. A. Micheelsen, and I. B. Dodd, "Ultrasensitive gene regulation by positive feedback loops in nucleosome modification," Molecular Systems Biology, vol. 4, no. 182, 2008.

[20] M. Ptashne, Genetic Switch: Phage Lambda Revisited. Cold Spring Harbor Laboratory Press, Cold Spring Harbor, NY, 2004.

[21] M. Acar, A. Becskei, and A. van Oudenaarden, "Enhancement of cellular memory by reducing stochastic transitions," Nature, vol. 435, 2005.

[22] J. Paulsson, "Summing up the noise in gene networks," Nature, vol. 427, pp. 415-418, 2004. 
[23] P. S. Swain, M. B. Elowitz, and E. D. Siggia, "Intrinsic and extrinsic contributions to stochasticity in gene expression," PNAS, vol. 99, pp. $12795-12800,2002$.

[24] J. Paulsson, "Model of stochastic gene expression," Physics of Life Reviews, vol. 2, pp. 157-175, 2005.

[25] V. Shahrezaei and P. S. Swain, "Analytical distributions for stochastic gene expression,” PNAS, vol. 105, pp. 17 256-17261, 2008.

[26] V. Shahrezaei, J. F. Ollivier, and P. S. Swain, "Colored extrinsic fluctuations and stochastic gene expression," Molecular Systems Biology, vol. 4, 2008.

[27] S. Hooshangi and R. Weiss, "The effect of negative feedback on noise propagation in transcriptional gene networks," CHAOS, vol. 16, 2006.

[28] L. Cai, C. K. Dalal, and M. B. Elowitz, "Frequency-modulated nuclear localization bursts coordinate gene regulation," Nature, vol. 455, pp. 485-490, 2008.

[29] D. Thieffry, A. M. Huerta, E. Prez-Rueda, and J. Collado-Vides, "From specific gene regulation to genomic networks: a global analysis of transcriptional regulation in escherichia coli," Bioessays, vol. 20, pp. 433-440, 1998.

[30] N. Rosenfeld, M. B. Elowitz, and U. Alon., "Negative autoregulation speeds the response times of transcription networks." J. Molecular Biology, vol. 323, pp. 785-793, 2002.

[31] M. Russel, L. Gold, H. Morrissett, and P. Z. O’Farrell, “Translational, autogenous regulation of gene 32 expression during bacteriophage t4 infection," J. of Biological Chemistry, vol. 251, pp. 7263-7270, 1976.

[32] T. Nogueira, M. de Smit, M. Graffe, and M. Springer, "The relationship between translational control and mrna degradation for the escherichia coli threonyl-trna synthetase gene," J. of Molecular Biology, vol. 310, pp. 709-722, 2001.

[33] M. H. A. Davis, Markov models and Optimization. Chapman and Hall, 1993.

[34] J. P. Hespanha, "Stochastic hybrid systems: Applications to communication networks," in Hybrid Systems: Computation and Control, ser. Lect. Notes in Comput. Science, R. Alur and G. J. Pappas, Eds. Berlin: Springer-Verlag, Mar. 2004, no. 2993 , pp. 387-401.

\section{APPENDIX A}

TRANSCRIPTIONAL NEGATIVE FEEDBACK

We model the time evolution of $z, m$ and $x$ through a Stochastic Hybrid System (SHS), the state of which is $y=[z, m, x]^{T}$. This SHS is characterized by trivial continuous dynamics

$$
\dot{y}=0,
$$


six reset maps $\phi_{i}(y)$

$$
\begin{aligned}
y \mapsto \phi_{1}(y)=\left[\begin{array}{c}
z+1 \\
m \\
x
\end{array}\right], \quad y \mapsto \phi_{2}(y)=\left[\begin{array}{c}
z-1 \\
m \\
x
\end{array}\right] \\
y \mapsto \phi_{3}(y)=\left[\begin{array}{c}
z \\
m+1 \\
x
\end{array}\right], \quad y \mapsto \phi_{4}(y)=\left[\begin{array}{c}
z \\
m-1 \\
z \\
m \\
x+1
\end{array}\right], \quad y \mapsto \phi_{6}(y)=\left[\begin{array}{c}
z \\
m \\
x-1
\end{array}\right] \\
y \mapsto \phi_{5}(y)=\left[\begin{array}{c}
z \\
x
\end{array}\right]
\end{aligned}
$$

and corresponding transition intensities

$$
\begin{aligned}
& \lambda_{1}(y)=k_{\text {exogenous }}, \lambda_{2}(y)=k_{\text {degexo }} z, \lambda_{3}(y)=k_{\text {transcription }} g_{1}(x, z), \\
& \lambda_{4}(y)=k_{\text {degmRNA }} m, \lambda_{5}(y)=k_{\text {translation }} m, \lambda_{6}(y)=k_{\text {deg pro }} x
\end{aligned}
$$

corresponding to exogenous noise production, exogenous noise degradation, transcription, translation, mRNA and protein degradation. The above SHS representation of the stochastic process is equivalent to the nonlinear Master equation, but from which it is easier to derive the differential equations for the statistical moments (see references [33], [34]). Note that except $\lambda_{3}(y)$ all other transition intensities are already linear. Linearizing $\lambda_{3}(y)$ about the steady-state levels we have

$$
\begin{aligned}
& \lambda_{3}(y)=k_{\text {transcription }} g_{1}(x, z)=\frac{g(z)}{1+(a x)^{H}} \\
& \approx k_{\text {transcription }} g_{1}(\bar{x}, \bar{z})\left[1+\left.\frac{d g(\bar{z})}{d z}\right|_{z=\bar{z}} \frac{z-\bar{z}}{g(\bar{z})}+\left.\frac{d g_{1}(x, \bar{z})}{d x}\right|_{x=\bar{x}} \frac{x-\bar{x}}{g_{1}(\bar{x}, \bar{z})}\right] .
\end{aligned}
$$

In the sequel we will use this linearized transition intensity to obtain the moment dynamics. The time evolution of the statistical moments is determined using the Dynkin's formula for the SHS (28)-(30), according to which, for every differentiable function $\psi(y)$ we have that

$$
\frac{d \mathbf{E}[\psi(\mathbf{y})]}{d t}=\mathbf{E}\left[\sum_{i=1}^{6}\left(\psi\left(\phi_{i}(\mathbf{y})\right)-\psi(\mathbf{y})\right) \lambda_{i}(\mathbf{y})\right]
$$


[33], [34]. Using (31), the time derivative of any vector $\mu$ defined by

$$
\mu=\left[\mathbf{E}[z], \mathbf{E}[m], \mathbf{E}[x], \mathbf{E}\left[z^{2}\right], \mathbf{E}\left[m^{2}\right], \mathbf{E}\left[x^{2}\right], \mathbf{E}[z m], \mathbf{E}[m x], \mathbf{E}[z x]\right]^{T}
$$

is given by

$$
\dot{\mu}=\overline{\mathbf{a}}+A \mu
$$

for an appropriate constant vector $\overline{\mathbf{a}}$ and a constant matrix $A$. A steady-state analysis of the above system of linear equations using Mathematica yields (8) and (10).

\section{APPENDiX B}

\section{TRANSLATIONAL NEGATIVE FEEDBACK}

The time evolution of the species count can modeled using a SHS similar to (28)-(30), with the only difference that the transition intensities are now given by

$$
\begin{aligned}
& \lambda_{1}(y)=k_{\text {exogenous }}, \lambda_{2}(y)=k_{\text {degexo }} z, \lambda_{3}(y)=k_{\text {transcription }} g(z), \\
& \lambda_{4}(y)=k_{\text {degmRNA }} m, \lambda_{5}(y)=k_{\text {translation }} m g_{2}(x), \lambda_{6}(y)=k_{\text {deg pro }} x .
\end{aligned}
$$

We linearize $\lambda_{5}(y)$ with respect to the means $\bar{m}, \bar{x}$ and approximate it as

$$
\lambda_{5}(y)=k_{\text {translation }} m g_{2}(x) \approx k_{\text {translation }} \bar{m} g_{2}(\bar{x})\left[\frac{m}{\bar{m}}+\left.\frac{d g_{2}(x)}{d x}\right|_{x=\bar{x}} \frac{x-\bar{x}}{g_{2}(\bar{x})}\right] .
$$

Performing the exact same analysis as in Appendix A, we have that the steady-state moments are given by (22) and (23). 


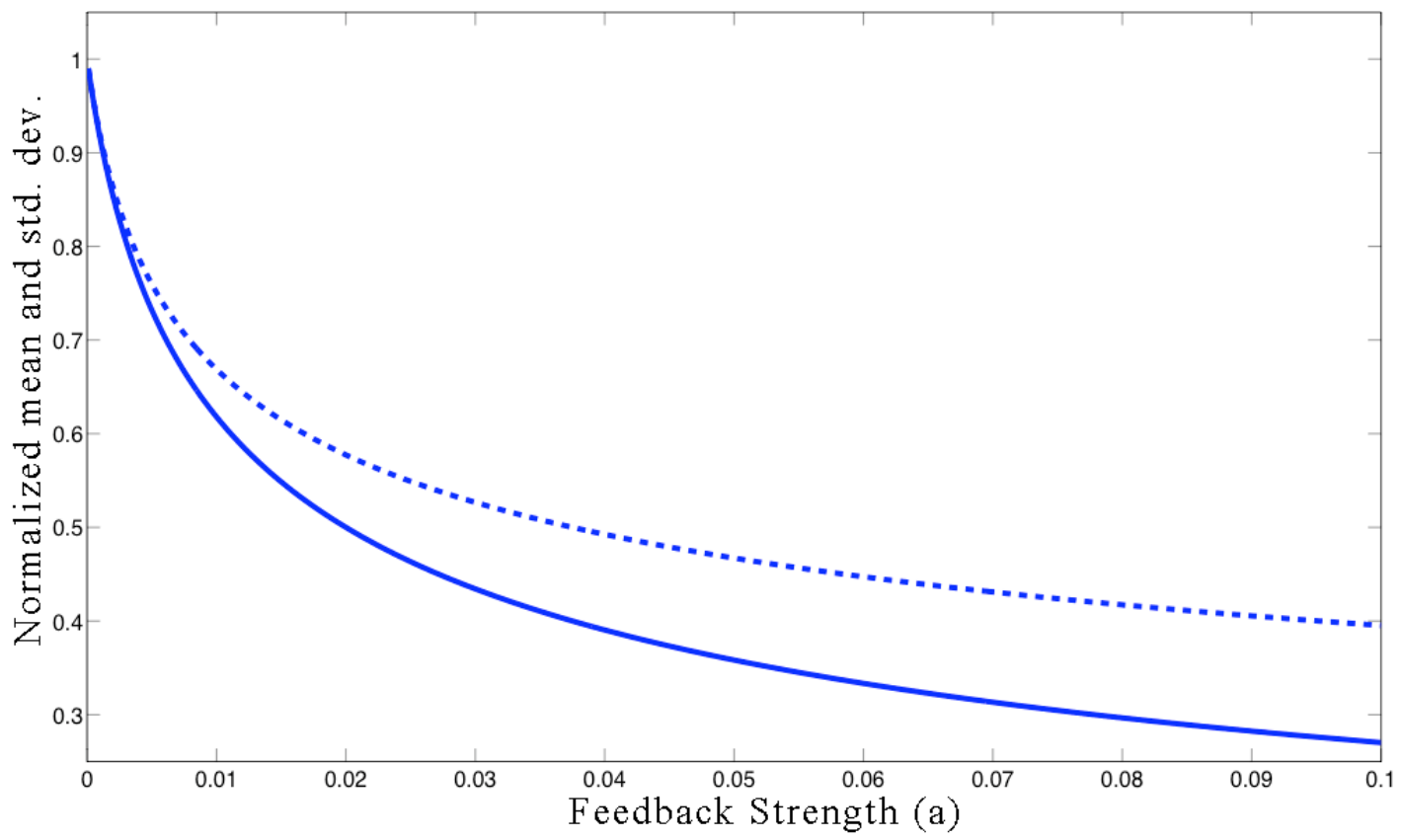

Fig. 1. The steady-state average protein count $\bar{x} / \bar{x}_{n f}$ (solid line) and the steady-state standard deviation $\sigma / \sigma_{n f}$ (dashed line) monotonically decreases with increasing negative feedback strength $a$. In this plot, both quantities are normalized by their corresponding values when there is no feedback (i.e., $a=0$ ). The other parameters used in this plot are $\bar{x}_{n f}=100, H=1, \sigma_{\text {exogenous }}=0$ and $\varepsilon \approx 0$. Note from (10) that when $\sigma_{\text {exogenous }}=0$ the ratio $\sigma / \bar{x} \propto 1 / \sqrt{\bar{x}}$. As $\bar{x}$ monotonically decreases with increasing negative feedback strength $a$, the ratio $\sigma / \bar{x}$ monotonically increases with $a$. 


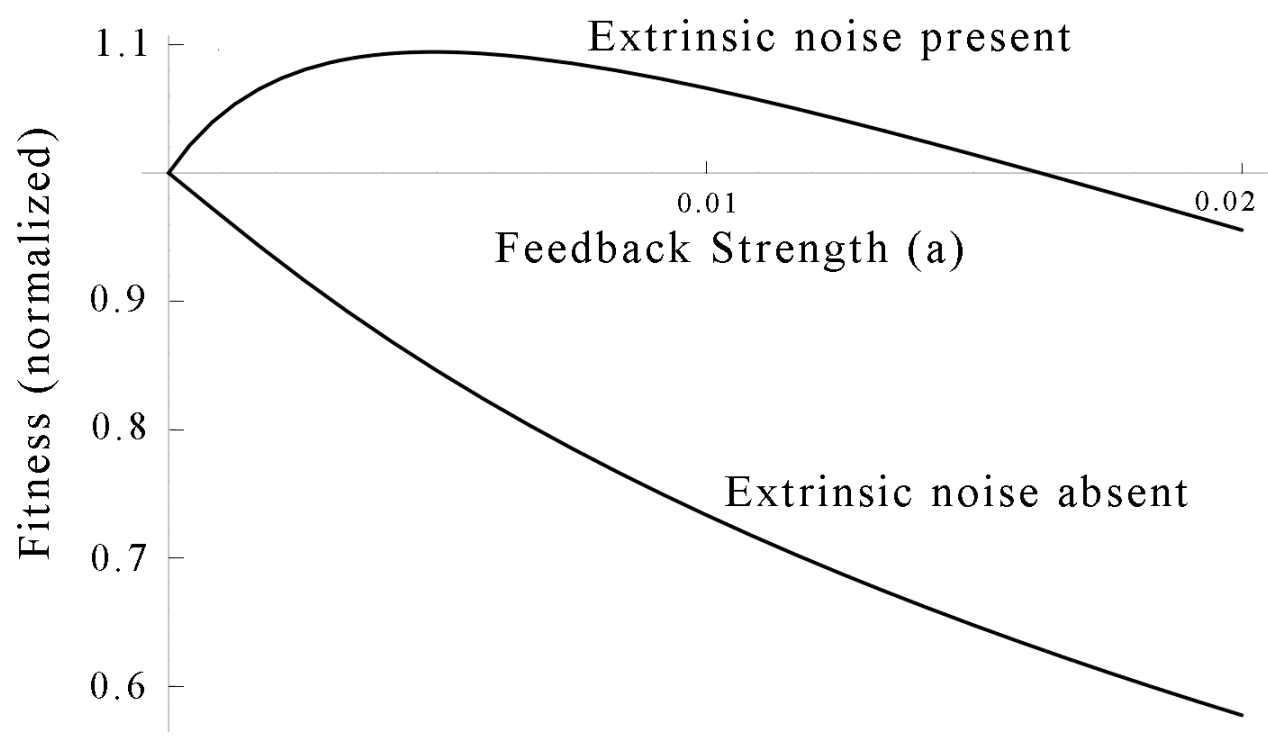

Fig. 2. The normalized fitness $F$ as a function of the feedback strength corresponding to a transcriptional feedback with $H=1$. When extrinsic noise is absent the fitness decreases with increasing $a$ (negative feedback is not evolvable). On the other hand, if there is significant extrinsic noise in the protein population, fitness increase with increasing $a$ near $a=0$ (negative feedback is evolvable). The other parameters used in this plot are $\gamma=1 / 3, \kappa \approx 0, \varepsilon \approx 0$ and $\bar{x}_{n f}=100$. 

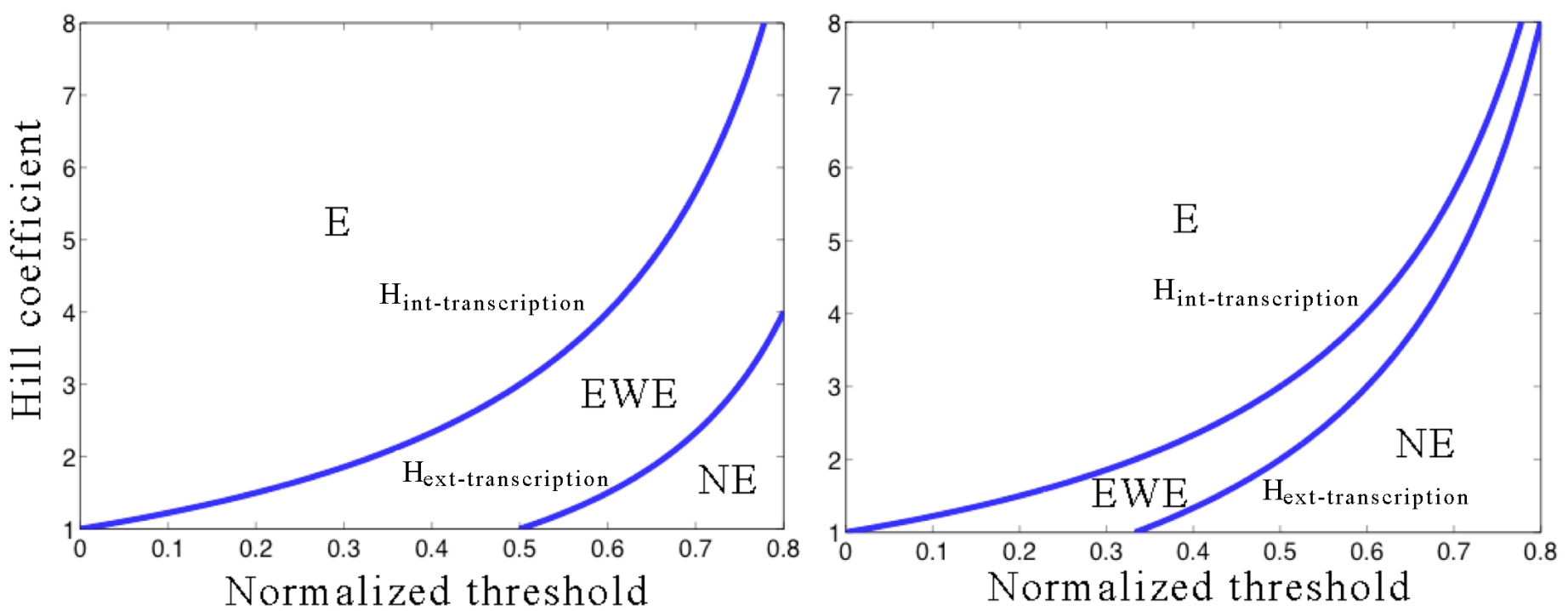

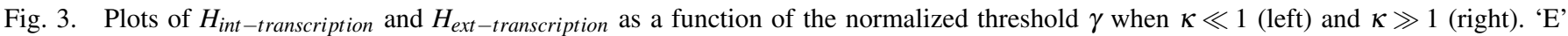
denotes the region $H>H_{\text {int-transcription }}$ where feedback is always evolvable irrespective of whether protein noise is intrinsic or extrinsic. This region is independent of $\kappa$. 'EWE' denotes the region $H_{\text {ext-transcription }}<H<H_{\text {int-transcription }}$ where feedback can only evolve if there is sufficiently large extrinsic noise in the protein count. This region shrinks with increasing $\kappa$. 'NE' denotes the region $H<H_{\text {ext-transcription }}$ where feedback is never evolvable. This region expands with increasing $\kappa$. For this and other plots we assume that the evolvability condition is given by (19), i.e., protein distribution is approximately Gaussian. 


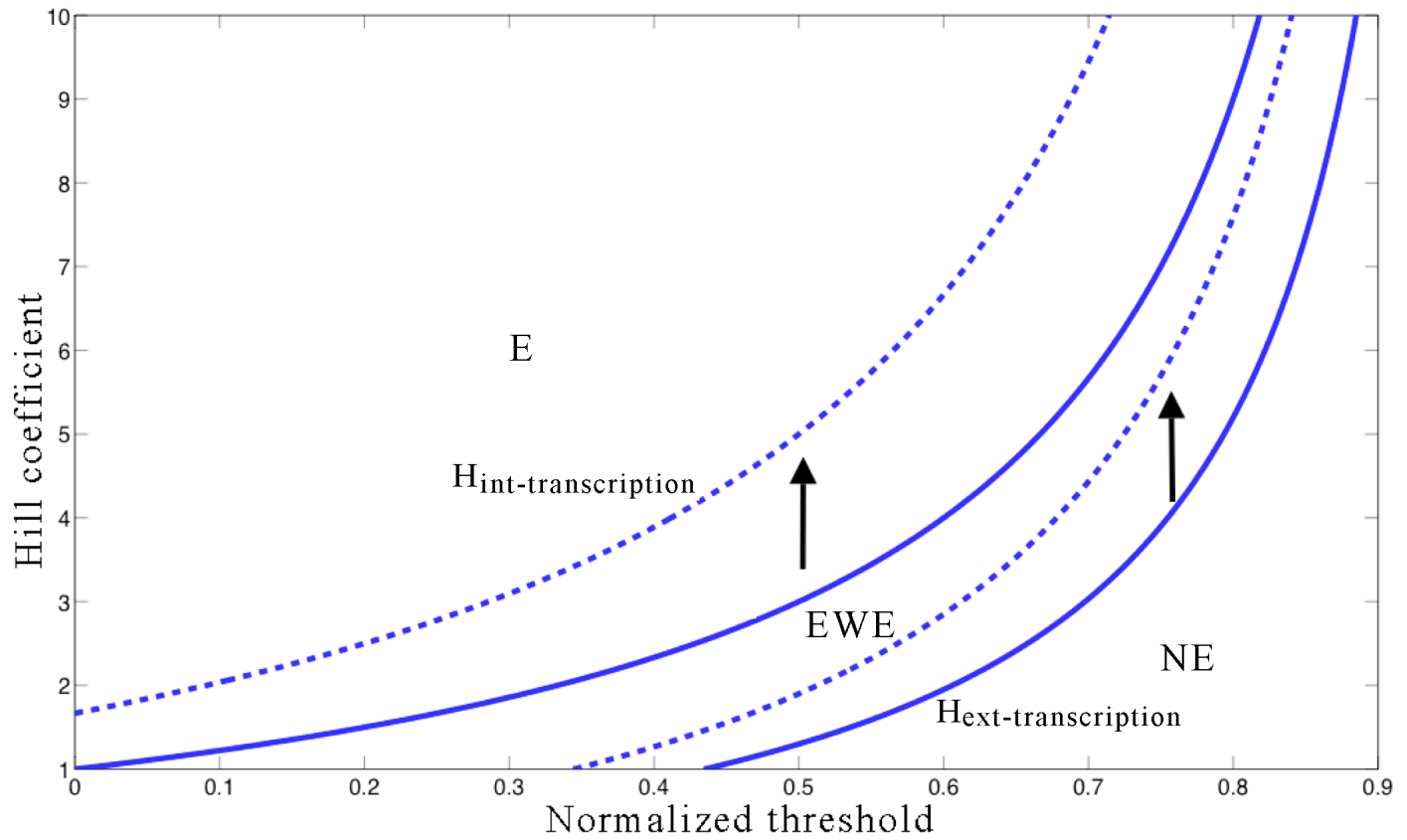

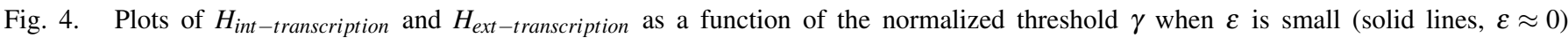
and $\varepsilon$ is large (dashed lines, $\varepsilon=5$ ). Both $H_{\text {int-transcription }}$ and $H_{\text {ext-transcription }}$ increase with increasing $\varepsilon$. As a result the evolvable region ('E'+'EWE') shrinks, while the non-evolvable region ('NE') expands with increasing $\varepsilon$. The other parameters used in this plot are $\kappa=\eta=1$. 


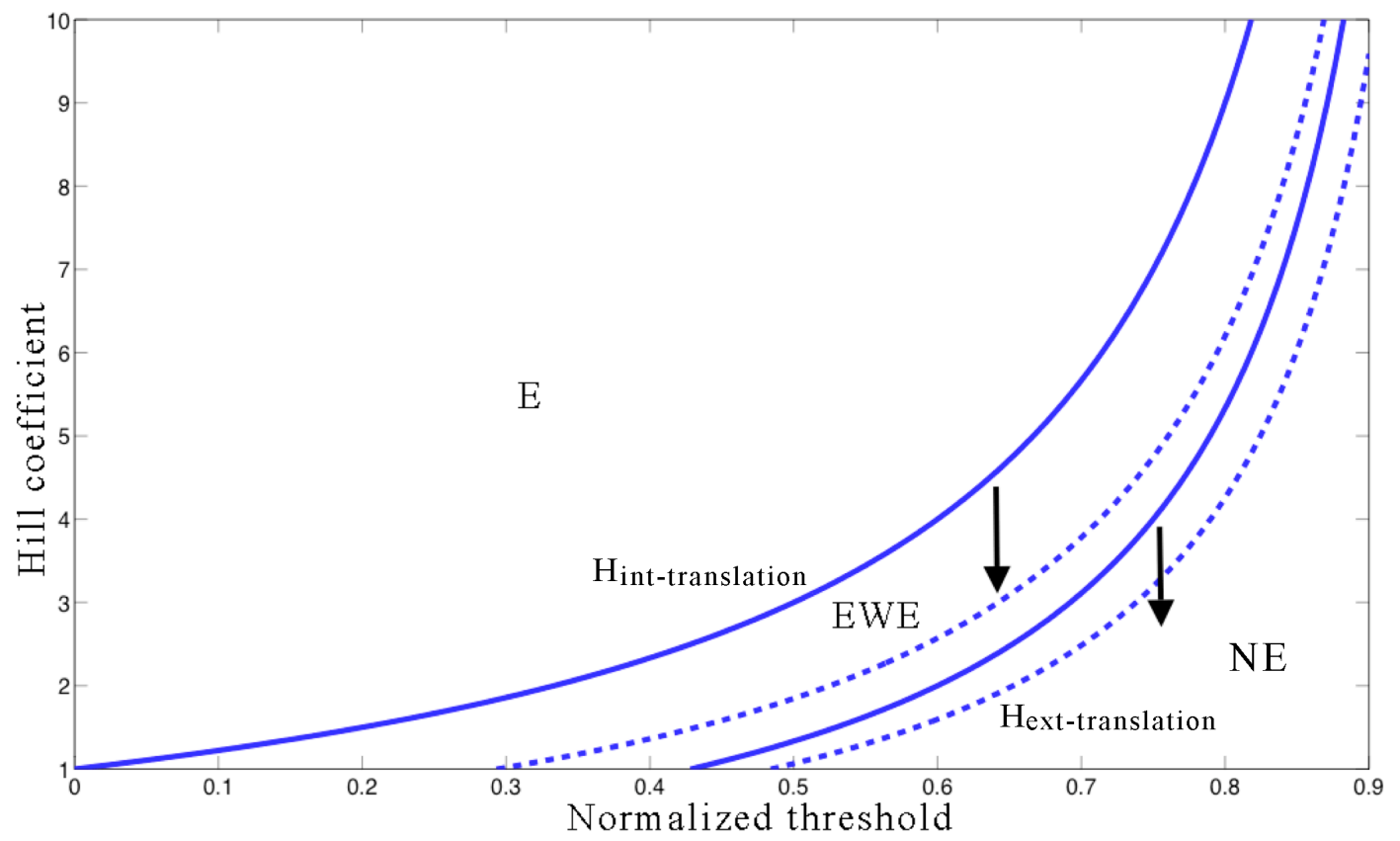

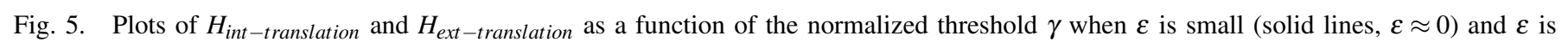
large (dashed lines, $\varepsilon=5$ ). Both $H_{\text {int-translation }}$ and $H_{\text {ext-translation }}$ decrease with increasing $\varepsilon$. As a result the evolvable region ('E'+'EWE') expands, while the non-evolvable region ('NE') shrinks with increasing $\varepsilon$. Other parameters as in Figure 5. 


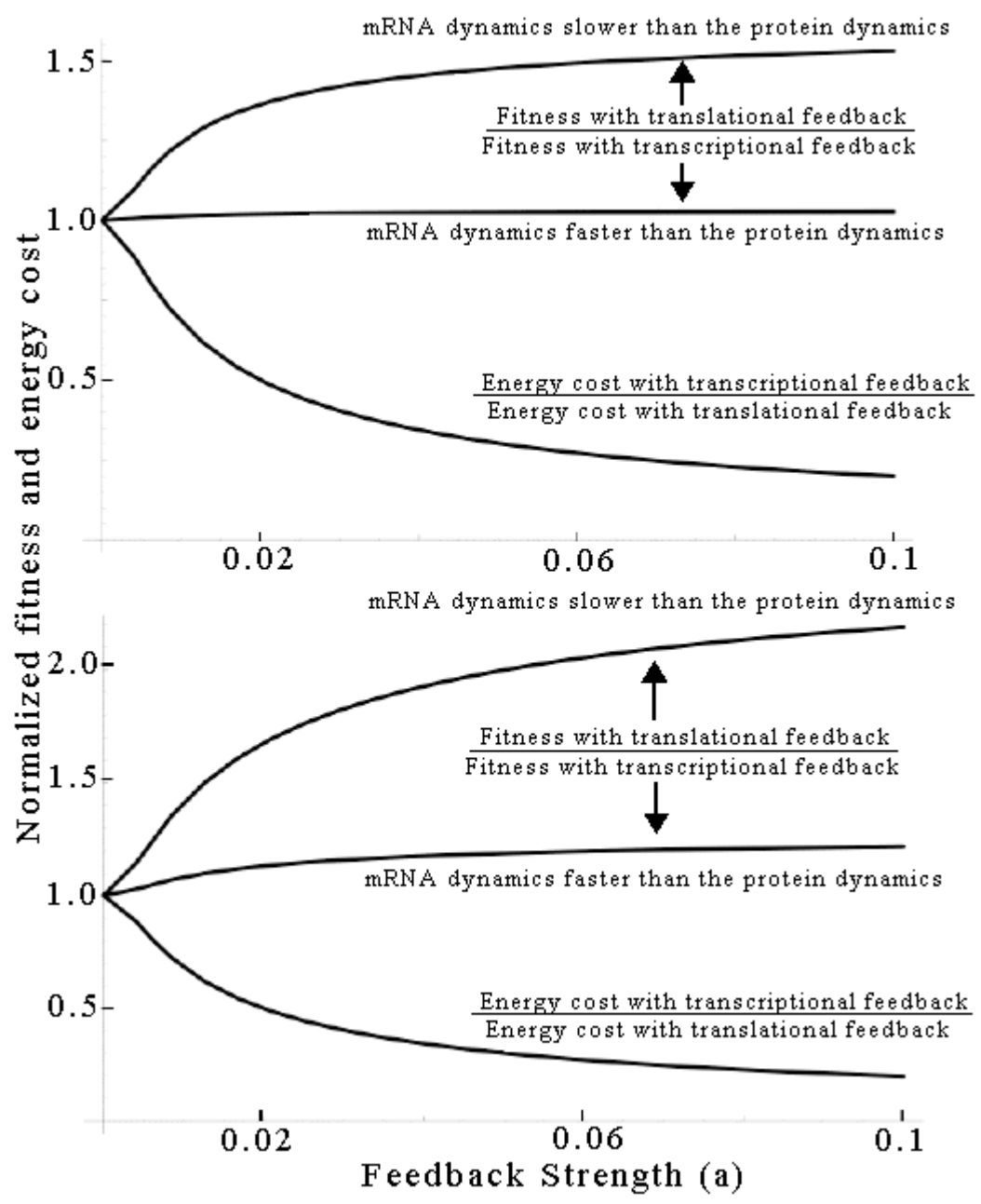

Fig. 6. The ratio of the normalized fitness $F$ and the steady-state energy expenditure (measured by the steady-state mRNA level) when $\sigma_{\text {int }} \ll \sigma_{\text {ext }}$ (top) and $\sigma_{\text {int }} \gg \sigma_{\text {ext }}$ (bottom) as a function of the negative feedback strength. mRNA dynamics faster (slower) than the protein dynamics corresponds to $\varepsilon=10(\varepsilon=0.1)$. Note from (8) and (22) that for a fixed $\bar{x}_{n f}$, the ratio of energy costs is independent of $\varepsilon$. The other parameters used in this plot are $\kappa=1, H=2, \eta=5, \gamma=1 / 3$ and $\bar{x}_{n f}=100$. 